\title{
Diffuse $\mathrm{H} \alpha$ in a Fractal Interstellar Medium
}

\author{
Bruce G. Elmegreen \\ IBM T. J. Watson Research Center, PO Box 218, \\ Yorktown Hts, NY 10598, USA \\ bge@watson.ibm.com \\ Received 1997 September 1, accepted 1997 November 3
}

\begin{abstract}
Observations of cloud structure over the last 10 years have led to the conclusion that it is fractal in nature, with a fractal dimension characteristic of turbulence, as found in the laboratory. Such structure has an open texture, with a volume filling factor of nearly $90 \%$ for gas that is at low density. Turbulence makes such cavities by clearing away material during convective motions. We propose that most of the low-density intercloud medium is the result of turbulence and not overlapping supernova remnants. This model has important implications for diffuse $\mathrm{H} \alpha$ and ionisation of the halo. Fractal clouds have a gradually decreasing average density with increasing distance, so ionisation zones around these clouds can be very extensive. Fractal clouds are also highly clustered, making the mean free path for ionising photons at least twice as large as in the 'standard cloud' model. This long mean free path allows stray ionising photons from midplane HII regions to reach and ionise the halo.
\end{abstract}

Keywords: turbulence - ISM: clouds - ISM: structure - H II regions

\section{Correlated Motions and Fractal Structures}

Interstellar gas is hierarchically clumped, with condensations and filaments inside larger, more tenuous condensations and filaments (Scalo 1985). This seems to be the case for all irregular structures that are not obvious shells or other disturbances (although even some shells are nested inside bigger shells). If we consider that the regular structures, like shells, spiral density waves, whole galaxy disks etc., are shaped directly by organised forces and respond on specific scales to boundary or initial conditions, then most of the interstellar structure that is far removed from such boundary forces is hierarchically clumped. This includes the lowdensity (undisturbed) gas between shells, the gas that lies inside the shell periphery (i.e. the swept-up gas), the gas inside spiral arms (as opposed to the arms themselves), and everywhere else that is free of confinement.

The origin of this pervasive hierarchical structure seems to be turbulence. Turbulence is characterised by correlated motions in which regions close to each other comove in space, and regions further apart move more independently (Larson 1981). Turbulence also has the property that there is no characteristic scale in many problems, aside from the molecular collision mean free path and the scale of the whole system, where the walls or other confinement structures occur. Scale-free structures are fractal (Mandelbrot 1983), so turbulent gas can become fractal far from its boundaries on a timescale comparable to the crossing time.

\section{Implications for Interstellar Clouds}

Fractal cloud structure showed up first in the form of fractal cloud edges (see reviews in Scalo 1990; Falgarone 1989; Zimmerman \& Stutzki 1993; Pfenniger 1996). This gave a projected fractal dimension equal to $1 \cdot 3$. Now fractal structure shows up in two other observations: the size distribution for clouds or clumps in CO surveys, and the mass size relation for clouds that are at about the same temperature (Elmegreen \& Falgarone 1996). This second study gives a fractal dimension for whole clouds equal to about $2 \cdot 3$. This dimension also applies to laboratory turbulence (Meneveau \& Sreenivasan 1990), and to such common structures as clouds in the Earth's atmosphere and smoke swirls. Interstellar clouds look like transparent atmospheric clouds because both get their structure from convected material undergoing turbulent motions. Elmegreen \& Falgarone (1996) also showed that the mass distribution function for interstellar clouds may result from turbulence.

If the fractal dimension of interstellar gas is $D$, then some basic correlations and distribution functions follow (Elmegreen 1997a; hereafter E97a):

1. A distribution function for structures of size $S$ :

$$
n(S) d \log S \propto S^{-D} d \log S .
$$

2. A mass size relation for clouds that have the same temperature: $M \propto S^{D}$ (temperature matters because the inferred $M$ is obtained from the cloud luminosity). 


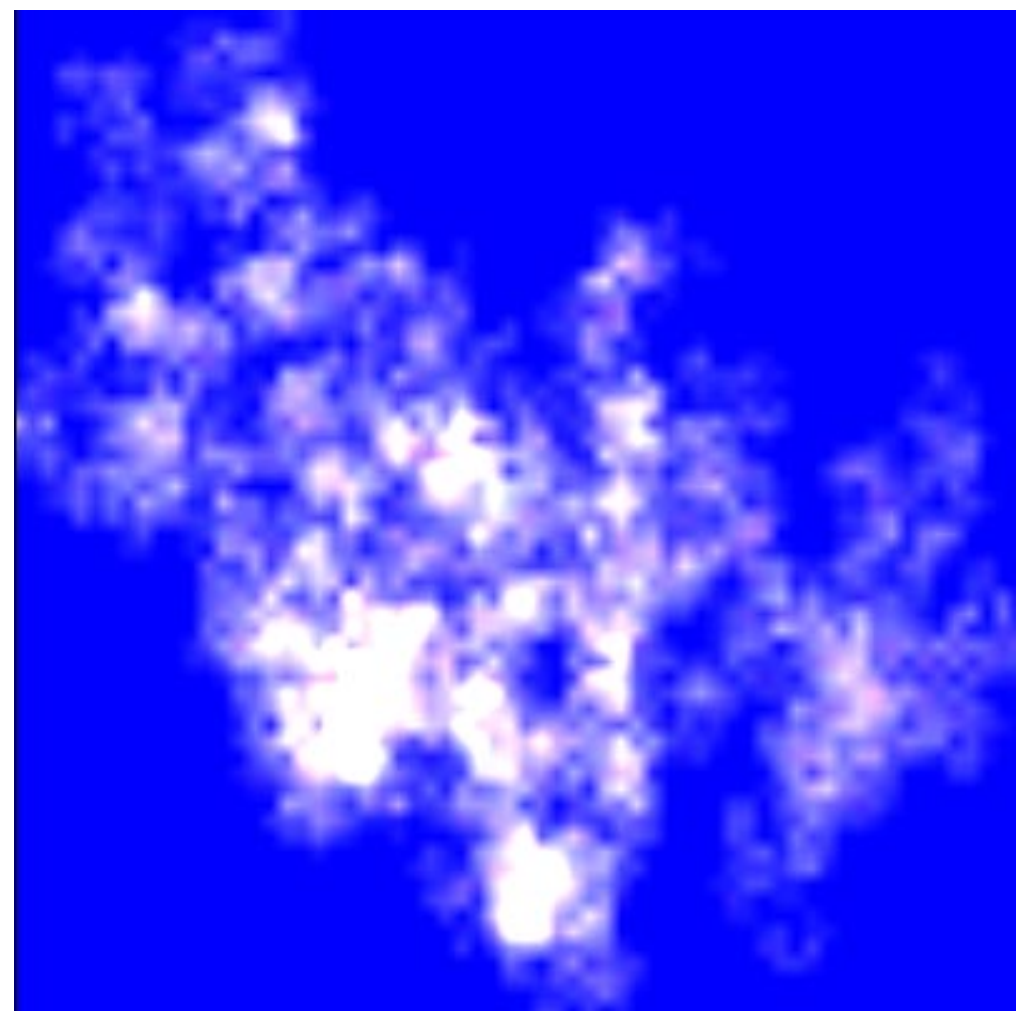

Figure 1-Model fractal cloud with $D=2 \cdot 3$.

3. A mass distribution function for clouds that have the same temperature: $n(M) d \log M \propto$ $M^{-1} d \log M$.

4. A mass distribution function for clouds that have an arbitrary mass-size relation, $M \propto S^{\kappa}$, giving $n(M) d \log M \propto M^{-D / \kappa} d \log M$.

5. A density mass relation: $\rho \propto M / S^{3} \propto M^{1-3 / D}$.

6. A density filling factor relation: $f \propto \rho^{-1}$.

7. A column-density $(\sigma)$, angular filling factor $\left(f_{\theta}\right)$ relation: $f_{\theta} \propto \sigma^{-1}$.

8. A distribution function for density: $n(\rho) d \rho \propto$ $\rho^{(3-2 D) /(D-3)} d \rho$.

For all of these relations, we should take $D \sim 2 \cdot 3$. Many properties of turbulent interstellar gas can be obtained from these relations, or conversely, $D$ can be measured from observed gas properties using them.

A fractal cloud with dimension $D=2 \cdot 3$ that is made from hierarchically clustered points is shown in Figure 1. Each point in the cluster is divided into $N=5$ other points, all within a distance $1 / L^{h}$ for hierarchical level $h$ and geometric factor $L=2$. To make it random, we choose random positions for these points within the distance $1 / L^{h}$. The point-clusters continue to subdivide down to $H=6$ total levels in the hierarchy, giving a maximum density contrast $C=L^{3 H}=2 \cdot 6 \times 10^{5}$ and a total number of points equal to $N^{H} \sim 1.5 \times 10^{4}$. The fractal dimension is defined to be $D=\log N / \log L$, which makes it $D \sim \log 5 / \log 2 \sim 2 \cdot 3$.
The resulting cloud is clumpy on a wide range of scales, like an interstellar cloud, and there are numerous empty regions inside it. The fraction of the total volume that is empty (the porosity) is given by

$$
f_{\text {empty }}=1-C^{(D / 3)-1}
$$

for maximum density contrast $C$ and fractal dimension $D$ (E97a). This is about $95 \%$ for the fractal in Figure 1 and slightly less for interstellar gas, since the maximum density contrast for either atomic or molecular regions is around $C \sim 10^{4}$. The fraction of the projected area of the cloud that is empty in Figure 1 is about $1 / 2$, as obtained from the distribution function of total empty area in numerous fractal models. We have proposed that such holes in the interstellar fractal produce much of the low-density intercloud medium, as reviewed in the next section.

\section{Intercloud Medium}

A turbulent interstellar medium should generate holes, tunnels and other empty structures as a result of fast clearing action by random supersonic motions. The origin of this energy might be supernova explosions (Norman \& Ferrara 1996), but the clearing can occur even far away from the explosions as the turbulent energy propagates around, perhaps on magnetic field lines. The source 
of turbulent energy might also be connected with galactic rotation because the $\mathrm{H}$ I velocity dispersion seems to remain fairly high even in the outer disks of some galaxies (Dickey, Hanson \& Helou 1990), where the star formation activity is low.

An analogy to the turbulent structures envisioned here might be with the surface of highly agitated water in a pan. The height of the water corresponds to the density of the gas in a compressible fluid. Regions of strong convergence in both the interstellar gas and the pan make dense or high peaks, and regions of strong divergence make holes or valleys, respectively. The resulting gradients then drive more motions and the compression and splashing create new structures elsewhere at a later time. This structuring continues as long as there is turbulent energy in the fluid.

There are several implications for the formation of random holes by turbulence. First, the McKee \& Ostriker (1977; 'MO') model of a supernovadominated ISM is not necessary and may even be inappropriate for several reasons (E97a):

1. Supernovae are not as large as MO proposed, and therefore they may not overlap, because the interstellar boundary pressure for each remnant is 10 times higher than MO assumed.

2. The OVI from hot gas is not pervasive but is confined to only a few regions per kpc (Shelton \& Cox 1994).

3. The filling factor of real shells is only around $10 \%$ in our Galaxy disk, not enough to fill the midplane intercloud medium with hot gas (Oey \& Clarke 1997).

4. Supernovae are highly clustered into OB associations because of the fractal cloud structure, so the rate of explosions that have access to low-density gas is much lower than MO assumed.

5. Most clouds have intricate small-scale structure along their periphery, much smaller than the lower limit of $1 \mathrm{pc}$ for the McKee-Ostriker clouds in a pervasive hot intercloud medium.

6. Clouds hit by a supernova shock are shredded and swept back (Klein, McKee \& Colella 1994), thereby absorbing the shock momentum. The radiation from the dense clouds also removes the supernova energy. Thus supernovae do not simply go around the clouds and expand into the pure intercloud medium, as the MO model assumed.

7. Supernova remnant shells are arched in a concave fashion; in the MO model, these visible shells were supposed to be only the shocked ambient clouds, while the actual hot remnant extended far beyond these shocked clouds. Shocked clouds are now known to be comet-shaped, however, with tails pointing outward, not arched parallel to the shock front. Such comets are observed in the Gum nebula, for example, but not generally in supernova shells.
A viable replacement for the McKee-Ostriker model is one based largely on turbulent motions, especially since the turbulent and fractal structure is observed almost everywhere anyway. Similar ideas were expressed by Passot, Vazquez-Semadeni \& Pouquet (1995), without specific concern for the fractal aspect that is emphasised here.

Of course, the turbulence interpretation for the intercloud medium would not apply to the holes inside giant shells and chimneys that surround massive star-formation sites - only to the low-density part of the ISM that is between these sites.

\section{Photon Mean Free Paths}

The clouds in a fractal interstellar medium are highly clumped, not uniformly dispersed as in the 'standard cloud' model. This clumping increases the mean free path for photons between the fractal cloud complexes, making the diffusion of photons easier than previously thought.

The change in photon mean free path can be understood from the fractal model in E97a, which gives the average number of subpieces on a line of sight through a fractal cloud complex. This number is $C^{(D-2) / 3} \sim 2.5$ for maximum density contrast $C$ and fractal dimension $D$. If the average number of clouds per kiloparsec is 8 , for example, and if each cloud complex contributes $2 \cdot 5$ absorption lines, then the average number of fractal cloud complexes per $\mathrm{kpc}$ is $8 / 2 \cdot 5=3$. The inter-complex mean free path is the inverse of this, or $0.3 \mathrm{kpc}$. This is so long that most photons that leak out of a midplane H II region can travel nearly to the next H II region or reach the halo. This produces extensive diffuse $\mathrm{H} \alpha$ that is directly associated with each H II region (see next section). It also allows the halo to be ionised by photons coming from the midplane.

This implication of a long intercloud mean free path is sensible considering the distribution of extinction in the solar neighbourhood. Lucke (1978) showed that the gas in the solar neighborhood is clumped into a few large diffuse cloud complexes, spaced by several hundred pc. These clouds produce the local interstellar absorption lines, such as those used by Blaauw (1952) to derive the average of 8 clouds/kpc. Thus, these 8 clouds per kpc are highly clumped in Lucke's map. Also, the mean separation between fractal cloud complexes derived from the model is about the same as the spacing between the Lucke clouds and between the OB associations/GMCs in the solar neighbourhood, including Orion, Perseus, ScoCen, Cepheus, etc. Essentially all of the local gas is known to be clumped into a few giant cloud complexes, which are generally composed of both atomic and molecular gas. Since we see the fractal structure of these local clouds directly, in CO and IRAS maps, there is direct evidence for the highly clumped and fractal structure that is emphasised in the new model. 
The intercloud medium is presumably the lowdensity part of the interstellar fractal. Another way of seeing why there must be a low-density part to the ISM in a turbulent model is by considering the density of thermally stable gas in the local radiation field. This density is in the range of 10 to $100 \mathrm{~cm}^{-3}$ for the cool phase, and much less, such as $0.1 \mathrm{~cm}^{-3}$, in the warm neutral phase, given the total interstellar pressure. The gas cannot remain stable at the average local density of $\sim 1 \mathrm{~cm}^{-3}$. When turbulence moves gas around, it produces a variety of structures and everywhere locally the gas temperature settles on a value that puts it in thermal equilibrium. The thermal properties of the gas are of secondary importance to this structure because the total pressure is strongly dominated by turbulence and magnetic fields. Thus the temperature is a slave to the dynamics (Vazquez-Semadeni, Passot \& Pouquet 1995; Elmegreen 1997b). The thermal properties determine the maximum density. This maximum is just the total pressure divided by $k T$. The point is that turbulence remains supersonic, and therefore highly compressive, because the stable thermal temperature for most of the gas has a sound speed that is much less than the average interstellar turbulent speed. As a result, most of the gas mass in the midplane has the density of a diffuse cloud or larger, and most of the gas occupies a volume filling factor equal to the average density divided by this diffuse cloud density, which is only a few per cent. There is no way to spread out the gas to have a uniform density equal to the average density when the cool thermal phase is both highly subsonic and denser than the average. Any random motion inevitably partitions the gas into clouds and an intercloud medium. What is new about the fractal interpretation is the recognition that such turbulence partitioning can lead to fractal structure with well determined and universal properties.

A numerical simulation of compressible hydromagnetic turbulence with enough spatial resolution to see correlated motions and fractal structure in a region that is far removed from the boundaries and sources of excitation is described in Elmegreen (1997b). This simulation is only one-dimensional, so many important aspects of real turbulence are not present.

\section{Fractal Ionisation Zones}

Fractal clouds have a gradually decreasing density with distance from the cloud centre, and this produces a smooth transition from the core of an H II region located in the cloud to the surrounding intercloud medium. Only along lines of sight that return to the cloud will there be sharply-defined edges to the H II region; elsewhere the H II region will gradually taper off. This extensive ionised gas can be the origin of the midplane diffuse $\mathrm{H} \alpha$ emission seen in our galaxy (Reynolds 1995) and other galaxies (Walterbos \& Braun 1994; Ferguson et al. 1996).

We may determine the fraction of the total ionisation that occurs outside the conventional Strömgren radius by the usual equation for detailed photon balance, but now with a density structure $n=n_{0}\left(R / R_{0}\right)^{D-3}$ for $D=2 \cdot 3$. We define $R_{0}$ to be the core radius of the HII region, and $R_{S}$ to be the conventional Strömgren radius at the core density $n_{0}$; this gives a total stellar UV luminosity of $(4 \pi / 3) \alpha n_{0}^{2} R_{S}^{3}$ for the recombination rate $\alpha$ to all but the ground state of $\mathrm{H}$. The ratio of the fractal ionisation radius to the Strömgren radius is (E97a):

$$
\frac{R_{I}}{R_{S}}=\frac{R_{0}}{R_{S}}\left[\frac{2 D-3}{3}\left(\frac{R_{S}^{3}}{R_{0}^{3}}-1\right)+1\right]^{1 /(2 D-3)} .
$$

The fraction of the total ionisation from an $\mathrm{O}$ star that is outside the conventional Strömgren radius is

$$
\begin{aligned}
f_{\text {outside }} & =\frac{\int_{R_{S}}^{R_{I}} n(R)^{2} 4 \pi R^{2} d R}{(4 \pi / 3) n^{2} R_{S}^{3}} \\
& =\frac{3}{2 D-3}\left(\frac{R_{S}}{R_{I}}\right)^{2 D-6}\left[\left(\frac{R_{I}}{R_{S}}\right)^{2 D-3}-1\right] .
\end{aligned}
$$

This fraction has the limiting value of 1 for large $R_{S} / R_{0}$, which corresponds to very intense uv radiation from an H II region core. For $R_{S} / R_{0}=4$, we get $R_{I} / R_{S}=2 \cdot 29$ and $f_{\text {outside }}=0 \cdot 74$. [Note that E97a had an error in this expression because the factor $3 /(2 D-3)$ was not included. The rest of the derivation there is correct, though.]

This result implies that a substantial amount of ionisation should leak out of H II region cores into the intercloud medium along lines of sight that pass through the tenuous fractal gas. The tiny clumps in this gas may be ionised and homogenised, but the overall density gradient should change only slowly.

\section{Conclusions}

Aside from a relatively low filling factor of interstellar matter that is in pressurised shells, and aside from any large-scale ordering in a spiral density wave or by 'beads-on-a-string' instabilities in such a wave, most of the ISM is hierarchically structured in a locally fractal fashion.

This structure shows up in the spatial distribution of young star ages, in the distribution function for cloud sizes and masses, in the correlation between cloud size and mass, and in the distribution function for star cluster mass (the cluster mass function was not discussed here, but see Elmegreen \& Efremov 1997). Fractal structure also shows up in the powerlaw relation between cloud perimeter and area.

The origin of the fractal structure is probably supersonic, sub-Alfvenic turbulence driven by a 
variety of pressure sources and possibly Galactic rotation. The fractal dimension of interstellar structure is about the same as the fractal dimension of laboratory turbulence.

These observations have far-reaching implications, some of which include:

1. The low-density intercloud medium could be generated partly by turbulence and partly by discrete pressure sources, such as supernovae remnants. The relative proportions of these two processes are not known but turbulence could dominate.

2. The interstellar gas is highly clumped, with $\sim 3$ fractal cloud complexes per kpc locally. Each complex has a hierarchical or fractal distribution of clumps within clumps.

3 . The extremely small-scale structure that is observed in diffuse clouds (Meyer \& Blades 1996; Watson \& Meyer 1996) and molecular clouds is probably the result of turbulence.

4. H II regions around $\mathrm{OB}$ associations extend 2 to 3 times further than previously believed, possibly accounting for much of the diffuse $\mathrm{H} \alpha$ in galactic disks.

5. The photon mean free path in the intercloud medium is $\sim 300 \mathrm{pc}$, allowing disk UV photons to reach the Galactic halo.

Blaauw, A. 1952, BAN, 11, 405

Dickey, J. M., Hanson, M. M., \& Helou, G. 1990, ApJ, 352, 522

Elmegreen, B. G. 1997a, ApJ, 477, 196 (E97a)

Elmegreen, B. G. 1997b, ApJ, 480, 674

Elmegreen, B. G., \& Efremov, Yu. 1997, ApJ, 480, 235
Elmegreen, B. G., \& Falgarone, E. 1996, ApJ, 471, 816

Falgarone, E. 1989, in Structure and Dynamics of the Interstellar Medium, ed. G. Tenorio-Tagle, M. Moles \& J. Melnick (Berlin: Springer), p. 68

Ferguson, A. M. N., Wyse, R. F. G., Gallagher, J. S. III., \& Hunter, D. A. 1996, AJ, 111, 2265

Klein, R. I., McKee, C. F., \& Colella, P. 1994, ApJ, 420, 213

Larson, R. B. 1981, MNRAS, 194, 809

Lucke, P. B. 1978, A\&A, 64, 367

McKee, C. F., \& Ostriker, J. P. 1977, ApJ, 218, 148

Mandelbrot, B. B. 1983, The Fractal Geometry of Nature (San Francisco: Freeman)

Meneveau, C., \& Sreenivasan, K. R. 1990, Phys. Rev. A, 41, 2246

Meyer, D. M., \& Blades, J. C. 1996, ApJ, 464, L179

Norman, C. A., \& Ferrara, A. 1996, ApJ, 467, 280

Oey, S., \& Clarke, C. 1997, MNRAS, 289, 570

Passot, T., Vazquez-Semadeni, E., \& Pouquet, A. 1995, ApJ, 455, 531

Pfenniger, D. 1996, in New Extragalactic Perspectives in the New South Africa, ed. D. L. Block \& J. M. Greenberg (Dordrecht: Kluwer), p. 439

Reynolds, R. J. 1995 in The Physics of the Interstellar Medium and Intergalactic Medium, ASP Conf. Ser. 80, ed. A. Ferrara, C. F. McKee, C. Heiles \& P. R. Shapiro (San Francisco: ASP), p. 388

Scalo, J. M. 1985 in Protostars and Planets II, ed. D. C. Black \& M. S. Mathews (Tucson: Univ. Arizona Press), p. 201

Scalo, J. M. 1990, in Physical Processes in Fragmentation and Star Formation, ed. R. Capuzzo-Dolcetta, C. Chiosi \& A. Di Fazio (Dordrecht: Kluwer), p. 151

Shelton, R. L., \& Cox, D. P. 1994, ApJ, 434, 599

Vazquez-Semadeni, E., Passot, T., \& Pouquet, A. 1995, in Fifth Mex-Tex Meeting in Astrophysics, Gaseous Nebula and Star Formation, ed. M. Peña \& S. Kurtz, Rev. Mex. Astron. Ap. Ser. Conf. 3, 61

Walterbos, R. A. M., \& Braun, R. 1994, ApJ, 431, 156

Watson, J. K., \& Meyer, D. M. 1996, ApJ, 473, L127

Zimmerman, T., \& Stutzki, J. 1993, Fractals, 1, 930 